\title{
ESSENTIALLY LARGE DIVISORS AND THEIR ARITHMETIC AND FUNCTION-THEORETIC INEQUALITIES*
}

\author{
GORDON HEIER ${ }^{\dagger}$ AND MIN RU ${ }^{\dagger}$
}

\begin{abstract}
Motivated by the classical Theorems of Picard and Siegel and their generalizations, we define the notion of an essentially large effective divisor and derive some of its arithmetic and function-theoretic consequences. We then investigate necessary and sufficient criteria for divisors to be essentially large. In essence, we prove that on a nonsingular irreducible projective variety $X$ with $\operatorname{Pic}(X)=\mathbb{Z}$, every effective divisor with $\operatorname{dim} X+2$ or more components in general position is essentially large.
\end{abstract}

Key words. Integral points, entire curves, hyperbolicity, Weil functions, Schmidt Subspace Theorem, Second Main Theorem.

AMS subject classifications. 11G35, 11G50, 14C20, 14G40, 32H30.

1. Introduction. In [CZ02], Corvaja-Zannier found an innovative way of using Schmidt's Subspace Theorem to give a new proof of the classical Theorem of Siegel on integral points on affine curves. They subsequently expanded their approach to obtain certain results on integral points in higher dimensions ([CZ03], [CZ04a], [CZ04b], [CZ06a],[CZ06b]). The approach was translated to Nevanlinna theory in [Ru04].

Ferretti and Evertse-Ferretti ([Fer00], [EF02], [EF08]) used similar (yet more general) arguments to obtain diophantine inequalities on projective varieties. Their approach is largely based on Mumford's degree of contact. In Nevanlinna theory, this approach was used by $\mathrm{Ru}$ [Ru09] to establish a Second Main Theorem for holomorphic curves into projective varieties intersecting hypersurfaces.

As stated in [Lev09, p. 609], the article [CZ02] motivated Levin to introduce the notions of large divisors and very large divisors as described in Definition 1.1. Their significance is due to the hyperbolicity-type and Mordell-type properties enjoyed by the complements of large divisors. Before we give the definition, a statement about the ground fields used seems in order. In this section, we let $K$ denote any field of characteristic zero. In Section 2, we will first assume $K=\mathbb{C}$ while dealing with the function-theoretic properties. Subsequently, we will take $K$ to be either a number field or the function field of a nonsingular projective variety over an algebraically closed field of characteristic 0. In Sections 3 and 4, we again take $K$ to be any field of characteristic zero. The term variety is taken to mean irreducible variety in this paper.

Definition 1.1 ([Lev09, Definition 8.1]). Let $D$ be an effective divisor on a nonsingular projective variety $X$ defined over $K$. Then $D$ is said to be very large (over $K$ ) if for every $P \in D(\bar{K})$, there exists a basis $B$ of the finite-dimensional vector space

$$
L(D)=\{f \text { rational function on } X \mid \operatorname{div}(f) \geq-D\}
$$

\footnotetext{
*Received July 16, 2011; accepted for publication August 31, 2011.

${ }^{\dagger}$ Department of Mathematics, University of Houston, 4800 Calhoun Road, Houston, TX 77204, USA (\{heier; minru\}@math.uh.edu). The first author was partially supported by the National Security Agency under Grant Number H98230-12-1-0235. The second author acknowledges partial support by NSA grants H98230-09-0004 and H98230-11-1-0201. The United States Government is authorized to reproduce and distribute reprints notwithstanding any copyright notation herein.
} 
such that $\operatorname{ord}_{E} \prod_{f \in B} f>0$ for every irreducible component $E$ of $D$ with $P \in E(\bar{K})$. Moreover, an effective divisor is said to be large if it has the same support as some very large divisor.

Recall that for an effective divisor $D$ on a nonsingular projective variety $X$ defined over $K$, the finite-dimensional vector spaces $L(D)$ and $H^{0}\left(X, \mathcal{O}_{X}(D)\right)$ are isomorphic via

$$
H^{0}\left(X, \mathcal{O}_{X}(D)\right) \rightarrow L(D), \quad s \mapsto \frac{s}{s_{D}},
$$

where $s_{D}$ is a section of $\mathcal{O}_{X}(D)$ with $\operatorname{div}\left(s_{D}\right)=D$.

For a divisor $D=\sum_{i=1}^{r} D_{i}$ with $D_{i}$ effective (but not necessarily irreducible), Levin's main sufficient criterion for very largeness is the following.

Lemma 1.2 ([Lev09, Lemma 9.1]). Let $D=\sum_{i=1}^{r} D_{i}$ be a divisor with each $D_{i}$ effective (but not necessarily irreducible) on a nonsingular projective variety $X$, all defined over $K$. For $P \in D(\bar{K})$, let $D_{P}=\sum_{\left\{i \mid P \in D_{i}\right\}} D_{i}$. Also, for integers $m$, n, let

$$
f_{P}(m, n)=h^{0}\left(X, \mathcal{O}_{X}\left(n D-m D_{P}\right)\right)-h^{0}\left(X, \mathcal{O}_{X}\left(n D-(m+1) D_{P}\right)\right) .
$$

If there exists $n>0$ such that $\sum_{m=0}^{\infty}(m-n) f_{P}(m, n)>0$ for all $P \in D(\bar{K})$, then $n D$ is very large.

The short proof provided in [Lev09] is based on the filtration argument introduced in [CZ02]. The main idea is to define a filtration

$$
V_{j}=H^{0}\left(X, \mathcal{O}_{X}\left(n D-j D_{P}\right)\right) \quad(j=0,1,2,3, \ldots)
$$

of $H^{0}\left(X, \mathcal{O}_{X}(n D)\right)$ and to choose a basis $f_{1}, \ldots, f_{h^{0}\left(X, \mathcal{O}_{X}(n D)\right)}$ of $L(n D)$ according to this filtration, beginning with the last nonzero subspace. Since $\operatorname{dim} V_{j} / V_{j+1}=$ $f_{P}(j, n)$, we get

$$
\operatorname{ord}_{E} \prod_{i=1}^{h^{0}\left(X, \mathcal{O}_{X}(n D)\right)} f_{i} \geq\left(\operatorname{ord}_{E} D\right) \sum_{m=0}^{\infty}(m-n) f_{P}(m, n)>0 .
$$

The main result on large divisors in [Lev09] is the following.

THEOREM 1.3 ([Lev09]). Let $X$ be a q-dimensional nonsingular projective variety, defined over $K$. Let $D=\sum_{i=1}^{r} D_{i}$ be a divisor, also defined over $K$, with each $D_{i}$ effective (but not necessarily irreducible) and big and nef. Moreover, assume that every irreducible component of $D$ is nonsingular and that the intersection of any $m+1$ distinct $D_{i}$ is empty over $\bar{K}$. If $r>2 m q$, then $D$ is large.

In the proof of this theorem given in [Lev09], the difference $h^{0}\left(X, \mathcal{O}_{X}(n D-\right.$ $\left.\left.m D_{P}\right)\right)-h^{0}\left(X, \mathcal{O}_{X}\left(n D-(m+1) D_{P}\right)\right)$ is bounded from above based on the $H^{0}$ part of the corresponding long exact cohomology sequence. As remarked by Levin, using the double filtration argument from [CZ04b, Lemma 3.2], the factor of 2 in the lower bound on $r$ can be removed if the $D_{i}, 1 \leq i \leq r$, do not have any irreducible components in common. See also [Aut09] and [CLZ09] for some more ideas in this direction.

It would of course be interesting to substantially decrease the lower bound on $r$ in Theorem 1.3. In light of the classical Theorems of Picard and Siegel and their generalizations, and some of the standard conjectures in hyperbolicity theory, one 
might suspect that when $m=q$, the lower bound of $2 m q=2 q^{2}$ can be replaced by $q+1$ (cf. Conjecture 1.10). However, it is quite clear that the sufficient criteria in [Lev09] cannot be used to prove this. To be precise, we make the following remark.

REMARK 1.4. In the situation of Lemma 1.2, let $X=\mathbb{P}^{q}$. Let $D$ be the sum of $r$ hypersurfaces in general position, i.e., the intersection of any $q+1$ of them is empty over $\bar{K}$. Let all hypersurfaces have the same degree. Then, the statement that, for all $P \in D(\bar{K})$,

$$
\sum_{m=0}^{\infty}(m-n) f_{P}(m, n)>0 \quad(n \text { sufficiently large })
$$

is equivalent to $r>q^{2}+q$.

We leave the proof of Remark 1.4, which is purely an exercise in handling binomial coefficients, to the reader. Note that since $\operatorname{Pic}\left(\mathbb{P}^{q}\right)$ is generated by $\mathcal{O}_{\mathbb{P} q}(1)$ and since $h^{0}\left(\mathbb{P}^{q}, \mathcal{O}_{\mathbb{P} q}(\ell)\right)=\left(\begin{array}{c}q+\ell \\ \ell\end{array}\right)$, the dimensions of all the $H^{0}$ 's involved are given explicitly.

We now turn to the new results presented in this note. The starting point is our definition of essentially (very) large divisors as follows.

Definition 1.5. Let $D$ be an effective divisor on a nonsingular projective variety $X$, all defined over $K$. Then we define $D$ to be essentially very large if there exists a linear subspace $V$ of $L(D)$ such that for every $P \in D(\bar{K})$, there exists a basis $B$ of $V$ such that $\operatorname{ord}_{E} \prod_{f \in B} f>0$ for every irreducible component $E$ of $D$ with $P \in E(\bar{K})$. Moreover, we define an effective divisor to be essentially large if it has the same support as some essentially very large divisor.

This is obviously a weakening of the notion of large divisors, which requires $V=$ $L(D)$. However, our definition seems technically easier to handle, while it retains the important arithmetic and function-theoretic consequences of the original definition of large divisors derived in [Lev09]. In fact, in Section 2, we prove new inequalities of Second Main Theorem-type and Schmidt Subspace Theorem-type for essentially large divisors that are actually stronger than the consequences for large divisors found in [Lev09]. Since the statements of our theorems require some (standard) definitions, we simply refer the reader to Theorem 2.2 and Theorem 2.10 at this point of the Introduction.

As a matter of convention, we will always assume that the constant function 1 is an element of the basis $B$, although it does not contribute to the vanishing of the product.

REMARK 1.6. The reader might wonder how essential very largeness fits in with the standard notions of "size" for a divisor in algebraic geometry. We only make the two following simple remarks in this respect.

First, it is clear from the definition that an irreducible divisor is never essentially large, regardless of how positive it is. In particular, even if it is very ample, it cannot be essentially large. Basically, essential largeness requires that the divisor be positive and have a sufficient number of irreducible components in general position. It is a simple exercise to see that on a smooth compact Riemann surface, an effective divisor is essentially large if and only if its support consists of three or more points.

Second, we remark that a basis for $V$ as in Definition 1.5 yields a nonconstant rational map to projective space whose locus of indeterminacy, i.e., the base locus of $V$ understood as a subspace of $H^{0}\left(X, \mathcal{O}_{X}(D)\right)$, is contained in the support of $D$. 
In a second line of inquiry, beginning in Section 3, it is clearly interesting to ask for (sharp) sufficient criteria for a divisor to be essentially large. In this direction, we first discovered that the filtration method from [CZ04a] (see also [Ru04]) yields the expected sharp bound (even for the original version of largeness) in the case of $X=\mathbb{P}^{q}$ as stated in the following theorem. (Cf. our comments in Remark 1.4.)

TheOREM 1.7. Let $q \geq 1$ and $r \geq q+2$ be integers. On $\mathbb{P}^{q}$, let $D=\sum_{i=1}^{r} D_{i}$ be a divisor defined over $K$, where each $D_{i}$ is a hypersurface (not necessarily irreducible or reduced). Assume that the $D_{i}$ are in general position, i.e., the intersection of any $q+1$ of them is empty over $\bar{K}$. Then $D$ is large.

In the case of arbitrary nonsingular projective varieties our result is the following.

THEOREM 1.8. Let $q \geq 1$ and $r \geq q+2$ be integers. Let $X \subseteq \mathbb{P}^{\ell}$ be a nonsingular projective variety of dimension $q$ defined over $K$. Let $D=\sum_{i=1}^{r} D_{i}$ be a divisor on $X$ such that each $D_{i}$ is defined by the restriction to $X$ of a homogeneous polynomial of degree $d_{i}$ in $K\left[X_{0}, \ldots, X_{\ell}\right]$. Finally, assume that the $D_{i}$ are in general position on $X$, i.e., the intersection of $X$ with any $q+1$ of them is empty over $\bar{K}$. Then $D$ is essentially large.

We remark that the assumptions in Theorem 1.8 correspond exactly to the current state-of-the-art for generalized geometric versions of the Second Main Theorem (see [Ru09]).

It seemed to be an interesting problem to find a broad class of projective varieties to which Theorem 1.8 can actually be applied. This is treated in Section 4 , where we obtain the following theorem, which is repeated as Corollary 4.1.

TheOREM 1.9. Let $q \geq 1$ and $r \geq q+2$ be integers. Let $X \subseteq \mathbb{P}^{\ell}$ be a nonsingular projective variety of dimension $q$, defined over $K$. Assume that $\operatorname{Pic}(X)=\mathbb{Z}$. Let $D=\sum_{i=1}^{r} D_{i}$ be an effective divisor on $X$ defined over $K$ such that the $D_{i}$ are in general position. Then $D$ is essentially large.

In closing this Introduction, we conjecture that the bound on the number of components in Theorem 1.9 is generally the correct one. Note that in this case, a positivity assumption clearly needs to be added; we suspect that bigness should suffice.

Conjecture 1.10. Let $q \geq 1$ and $r \geq q+2$ be integers. Let $X \subseteq \mathbb{P}^{\ell}$ be a nonsingular projective variety of dimension $q$, defined over $K$. Let $D=\sum_{i=1}^{r} D_{i}$ be an effective divisor, defined over $K$, on $X$ such that the $D_{i}$ are big and in general position. Then $D$ is essentially large.

Acknowledgement. We thank the anonymous referees for their careful reading of our manuscript and their helpful suggestions, in particular concerning the case of function fields.

\section{The arithmetic and function-theoretic properties of essentially large} divisors.

2.1. The function-theoretic case. We first derive an inequality of Second Main Theorem-type for essentially very large divisors, where we use the definition of the essential (very) largeness with $K=\mathbb{C}$. We begin by recalling some standard definitions from Nevanlinna Theory.

Let $g: \mathbb{C} \rightarrow \mathbb{P}^{m}$ be a holomorphic map. Let $g=\left[g_{0}, \ldots, g_{m}\right]$ be a reduced representative of $g$, where $g_{0}, \ldots, g_{m}$ are entire functions on $\mathbb{C}$ and have no common 
zeros. The Nevanlinna-Cartan characteristic function (or the height function) $T_{g}(r)$ is defined by

$$
T_{g}(r)=\frac{1}{2 \pi} \int_{0}^{2 \pi} \log \max _{j=0, \ldots, m}\left|g_{j}\left(r e^{\sqrt{-1} \theta}\right)\right| d \theta .
$$

The above definition is independent, up to an additive constant, of the choice of the reduced representation of $g$.

For a divisor $D$ on a projective variety $X$, represented by a local defining function $\rho$, and a holomorphic map $g: \mathbb{C} \rightarrow X$, the counting function is defined as

$$
N_{g}(r, D)=\int_{0}^{r} \frac{n_{g}(t, D)-n_{g}(0, D)}{t} d t+n_{g}(0, D) \log r,
$$

where $n_{g}(t, D)$ is the number of zeros of $\rho \circ g$ inside $\{|z|<t\}$ (counting multiplicities), and $n_{g}(0, D)=\lim _{t \rightarrow 0^{+}} n_{g}(t, D)$.

By the Jensen formula, we also have

$$
N_{g}(r, D)=\sum_{a \in D_{r} \backslash\{0\}} \operatorname{ord}_{a}(\rho \circ g) \log \left|\frac{r}{a}\right|+\operatorname{ord}_{0}(\rho \circ g) \log r .
$$

The following generalized version of Cartan's Second Main Theorem (see [Ru97], [Voj97]) will be the basis of the proof of Theorem 2.2. Note that by \|| $\|$ we mean the (coefficient-wise) maximum norm in this subsection.

Theorem 2.1. Let $f=\left[f_{0}: \ldots: f_{m}\right]: \mathbb{C} \rightarrow \mathbb{P}^{m}$ be a holomorphic map whose image is not contained in a proper linear subspace. Let $H_{1}, \ldots, H_{q}$ be arbitrary hyperplanes in $\mathbb{P}^{m}$. Let $L_{j}, 1 \leq j \leq q$, be linear forms defining $H_{1}, \ldots, H_{q}$. Then, for every $\varepsilon>0$,

$$
\int_{0}^{2 \pi} \max _{\mathcal{K}} \log \prod_{j \in \mathcal{K}} \frac{\left\|f\left(r e^{\sqrt{-1} \theta}\right)\right\|\left\|L_{j}\right\|}{\mid L_{j}\left(f\left(r e^{\sqrt{-1} \theta}\right) \mid\right.} \frac{d \theta}{2 \pi} \cdot \leq .(m+1+\varepsilon) T_{f}(r),
$$

where ". $\leq$. " means that the inequality holds for all $r$ outside of a set $\Gamma$ with finite Lebesgue measure, and the maximum is taken over all subsets $\mathcal{K}$ of $\{1, \ldots, q\}$ such that the linear forms $L_{j}, j \in \mathcal{K}$, are linearly independent.

To prove our result, we need a general formula for the height function $T_{g}(r)$ when $g=\left[g_{0}: \ldots: g_{m}\right]$, where $g_{0}, \ldots, g_{m}$ are meromorphic functions (i.e., it is not necessarily the reduced representation of $g$ ). According to [Lan87, p. 202], such a formula reads

$$
\begin{aligned}
T_{g}(r)= & \int_{0}^{2 \pi} \log \max _{j=0, \ldots, m}\left|g_{j}\left(r e^{\sqrt{-1} \theta}\right)\right| \frac{d \theta}{2 \pi}-\log \max _{j \in A}\left|c_{g_{j}}\right| \\
& +\sum_{a \in D_{r} \backslash 0} \max _{j=0, \ldots, m}\left(-\operatorname{ord}_{a}\left(g_{j}\right)\right) \log \left|\frac{r}{a}\right|+\max _{j=0, \ldots, m}\left(-\operatorname{ord}_{0}\left(g_{j}\right)\right) \log |r|,
\end{aligned}
$$

where $\log \max _{j \in A}\left|c_{g_{j}}\right|$ is a correction term that makes the definition independent of multiplication by a nowhere zero holomorphic function (see [Lan87] for full details).

Our main result in this subsection is the following inequality of Second Main Theorem-type. According to the subsequent Corollary 2.3, it can be understood from the geometric point of view as a quantitative version of the results about the 
degeneracy of holomorphic mappings omitting essentially large divisors (see Corollary $2.3)$.

TheOREM 2.2. Let $D$ be an essentially very large divisor on a nonsingular complex projective variety $X$. Let $V$ be a linear subspace of $L(D)$ as in Definition 1.5. Let $\phi_{0}, \ldots \phi_{m}$ be an arbitrary basis for $V$. Let $f: \mathbb{C} \rightarrow X$ be an algebraically nondegenerate holomorphic map. Let $\Phi$ be the rational map $\left[\phi_{0}: \ldots: \phi_{m}\right]: X \rightarrow \mathbb{P}^{m}$, and write

$$
F=\Phi \circ f: \mathbb{C} \rightarrow \mathbb{P}^{m}
$$

When $E_{1}, \ldots, E_{\ell}$ denote the irreducible components of $D$, let

$$
M=\max \left\{-\operatorname{ord}_{E_{i}}\left(\phi_{j}\right) \mid i=1, \ldots, \ell, j=0, \ldots, m\right\} .
$$

Then, for every $\varepsilon>0$,

$$
T_{F}(r) . \leq .(M(m+1)+1+\varepsilon) N_{f}(r, D),
$$

where ". $\leq . "$ means that the inequality holds for all $r$ outside of a set $\Gamma$ with finite Lebesgue measure.

Note that since $\Phi$ is nonconstant and $f$ is algebraically nondegenerate, the map $F$ also is nonconstant.

Corollary 2.3. Let $D$ be an essentially large divisor on a nonsingular complex projective variety $X$. Then every holomorphic map $f: \mathbb{C} \rightarrow X \backslash D$ must be algebraically degenerate, i.e., the image of $f$ must be contained in a proper subvariety of $X$.

Proof of Corollary 2.3. Since the statement of the corollary only refers to the support of $D$, we can assume that $D$ is essentially very large. Assume that $f: \mathbb{C} \rightarrow$ $X \backslash D$ is algebraically nondegenerate. Since $f$ omits $D, N_{f}(r, D)=0$. So the above inequality implies that $T_{F}(r)$ is bounded outside of a set of finite Lebesgue measure. However, since $F$ is nonconstant, this is false and gives a contradiction.

Proof of Theorem 2.2. It is easy to see ([Lev09, Remark 8.2]) that there exists a finite set $J$ of elements in $V$ such that for every $P \in D$ there exists a subset $I \subset J$ that is a basis of $V$ with $\operatorname{ord}_{E} \prod_{f \in I} f>0$ when $E$ is a component of $D$ with $P \in E$. Let $J^{\prime}$ be the set of linear forms $L$ in $m+1$ variables such that $L \circ \Phi \in J$.

According to Theorem 2.1,

$$
\int_{0}^{2 \pi} \max _{I} \log \prod_{L \in I} \frac{\left\|F\left(r e^{\sqrt{-1} \theta}\right)\right\|\|L\|}{\left|L\left(F\left(r e^{\sqrt{-1} \theta}\right)\right)\right|} \frac{d \theta}{2 \pi} \cdot \leq \cdot(m+1+\varepsilon) T_{F}(r)
$$

where the maximum is taken over subsets $I \subset J^{\prime}$ such that $I$ consists of exactly $m+1$ independent linear forms.

Since the left-hand side is independent of the choice of the representation of $F$, we can rewrite the above inequality as

$$
\int_{0}^{2 \pi} \max _{I} \log \prod_{L \in I} \frac{\max _{0 \leq j \leq m}\left|\phi_{j}\left(f\left(r e^{\sqrt{-1} \theta}\right)\right)\right|}{\left|L\left(F\left(r e^{\sqrt{-1} \theta}\right)\right)\right|} \frac{d \theta}{2 \pi} . \leq .(m+1+\varepsilon) T_{F}(r)+O(1),
$$

where $O(1)$ is introduced by dropping the factor $\|L\|$ after assuming that a fixed choice of coefficients has been made. 
Next, we observe that since $D$ is essentially very large, outside of the support of the divisor $D$, we have

$$
\max _{j}\left|\phi_{j}\left(f\left(r e^{\sqrt{-1} \theta}\right)\right)\right|^{\frac{1}{M}} \min _{I} \prod_{L \in I}\left|L\left(F\left(r e^{\sqrt{-1} \theta}\right)\right)\right| \leq C,
$$

where $C$ is some constant. The reason is that we can cover $X$ by a finite number of open sets on each of which $\left|\phi_{j} \min _{I}\left(\prod_{L \in I}|L \circ \Phi|\right)^{M}\right|$ is bounded for each $j=0, \ldots, m$. Namely, for $P \in X$, define $U_{P}$ as follows. If $P \notin D$, take a small neighborhood $U_{P}$ of $P$ such that its closure is disjoint from the support of $D$. Since the $\phi_{j}$ have no poles outside of the support of $D$, the boundedness on $U_{P}$ is obvious. If $P \in D$, take $U_{P}$ to be a small neighborhood of $P$ with the property that $Q \in E \cap U_{P}$ implies $P \in E$ for all components $E$ of $D$. Consequently, by the essential very largeness, there exists $I_{0}$ such that $\operatorname{ord}_{E}\left(\prod_{L \in I_{0}} L \circ \Phi\right)$ is a positive integer and $\operatorname{ord}_{E}\left(\phi_{j} \min _{I}\left(\prod_{L \in I}|L \circ \Phi|\right)^{M}\right) \geq 0$ for all $j=0, \ldots, m$. Perhaps after shrinking $U_{P}$, this implies that $\left|\phi_{j} \min _{I}\left(\prod_{L \in I}|L \circ \Phi|\right)^{M}\right|$ is bounded on $U_{P}$ for every $j=0, \ldots, m$. Finally, since $X$ is compact, we can cover it with a finite number of open sets of the form $U_{P}$.

Applying log to both sides of (3) yields

$$
\log \max _{j}\left|\phi_{j}\left(f\left(r e^{\sqrt{-1} \theta}\right)\right)\right|^{\frac{1}{M}}+\log \min _{I} \prod_{L \in I}\left|L\left(F\left(r e^{\sqrt{-1} \theta}\right)\right)\right| \leq \log C .
$$

Therefore,

$$
\log \max _{j}\left|\phi_{j}\left(f\left(r e^{\sqrt{-1} \theta}\right)\right)\right|^{m+1+\frac{1}{M}} \leq \max _{I} \log \prod_{L \in I} \frac{\max _{j}\left|\phi_{j}\left(f\left(r e^{\sqrt{-1} \theta}\right)\right)\right|}{\left|L\left(F\left(r e^{\sqrt{-1} \theta}\right)\right)\right|}+\log C .
$$

Thus

$$
\begin{gathered}
\int_{0}^{2 \pi} \log \max _{j}\left|\phi_{j}\left(f\left(r e^{\sqrt{-1} \theta}\right)\right)\right| d \theta \\
=\frac{M}{M(m+1)+1} \int_{0}^{2 \pi} \log \max _{j}\left|\phi_{j}\left(f\left(r e^{\sqrt{-1} \theta}\right)\right)\right|^{m+1+\frac{1}{M}} d \theta \\
\leq \frac{M}{M(m+1)+1}\left(\int_{0}^{2 \pi} \max _{I} \log \prod_{L \in I} \frac{\max _{j}\left|\phi_{j}\left(f\left(r e^{\sqrt{-1} \theta}\right)\right)\right|}{\left|L\left(F\left(r e^{\sqrt{-1} \theta}\right)\right)\right|} d \theta+\log C\right) \\
\cdot \leq \cdot \frac{M(m+1+\varepsilon)}{M(m+1)+1} T_{F}(r)+O(1) .
\end{gathered}
$$

On the other hand, from (2),

$$
\begin{aligned}
T_{F}(r)= & \int_{0}^{2 \pi} \log \max _{j}\left|\phi_{j}(f)\left(r e^{\sqrt{-1} \theta}\right)\right| \frac{d \theta}{2 \pi}-\log \max _{j \in K}\left|c_{\phi_{j}(f)}\right| \\
& +\sum_{a \in D_{r} \backslash 0} \max _{j}\left(-\operatorname{ord}_{a}\left(\phi_{j}(f)\right)\right) \log \left|\frac{r}{a}\right| \\
& +\max _{j}\left(-\operatorname{ord}_{0}\left(\phi_{j}(f)\right)\right) \log |r| .
\end{aligned}
$$

Thus,

$$
\begin{aligned}
T_{F}(r) . \leq & \frac{M(m+1+\varepsilon)}{M(m+1)+1} T_{F}(r)+\sum_{a \in D_{r} \backslash 0} \max _{j}\left(-\operatorname{ord}_{a}\left(\phi_{j}(f)\right)\right) \log \left|\frac{r}{a}\right| \\
& +\max _{j}\left(-\operatorname{ord}_{0}\left(\phi_{j}(f)\right)\right) \log |r|+O(1) .
\end{aligned}
$$


Recall that due to the isomorphism (1), there are sections $s_{0}, \ldots, s_{m} \in H^{0}\left(X, \mathcal{O}_{X}(D)\right)$ such that $\phi_{j}=\frac{s_{j}}{s_{D}}$. Therefore, for all $a \in D_{r}$,

$$
\begin{aligned}
\max _{j}\left(-\operatorname{ord}_{a}\left(\phi_{j} \circ f\right)\right) & =\max _{j}\left(-\operatorname{ord}_{a}\left(\frac{s_{j}}{s_{D}} \circ f\right)\right) \\
& =\operatorname{ord}_{a}\left(s_{D} \circ f\right)-\min _{j} \operatorname{ord}_{a}\left(s_{j} \circ f\right) \leq \operatorname{ord}_{a}\left(s_{D} \circ f\right) .
\end{aligned}
$$

Consequently, we obtain

$$
\begin{aligned}
\frac{1-\varepsilon}{M(m+1)+1} T_{F}(r) . \leq & \sum_{a \in D_{r} \backslash\{0\}} \operatorname{ord}_{a}\left(s_{D} \circ f\right) \log \left|\frac{r}{a}\right| \\
& +\operatorname{ord}_{0}\left(s_{D} \circ f\right) \log r+O(1) \\
= & N_{f}(r, D)+O(1) .
\end{aligned}
$$

Note that since $T_{F}(r) \rightarrow \infty$ for $r \rightarrow \infty$, we have, after perhaps enlarging $\Gamma$, $N_{f}(r, D)+O(1) . \leq . N_{f}(r, D)+\delta T_{F}(r)$, where $\delta$ is any positive number. We can now subtract $\delta T_{F}(r)$ from both sides of the inequality and then divide both sides by $\frac{1-\varepsilon}{M(m+1)+1}-\delta$. Choosing small enough $\varepsilon$ and $\delta$ (depending on $\varepsilon$ ) concludes the proof. $\square$

2.2. The arithmetic case. We now derive an inequality of Schmidt Subspace Theorem-type for essentially large divisors. In this subsection, we work in the setting where $K$ is either a number field $k$, i.e., a finite extension of the field of rational numbers, or a function field $L$ of a nonsingular projective variety over an algebraically closed field of characteristic 0 . We write $K$ when referring to both cases at the same time. We remark that both types of fields are examples of global fields of characteristic zero with a proper set of absolute values $M_{K}$ such that the crucial product formula holds (see [Lan83, Chapters 1, 2]).

We begin with the case of a number field $k$ of degree $\mathfrak{d}$. We denote by $M_{k}$ the set of places (i.e., equivalence classes of absolute values) of $k$ and write $M_{k}^{\infty}$ for the set of archimedean places of $k$. In an archimedean class $v$, we choose the absolute value ||$_{v}$ such that ||$_{v}=||$ on $\mathbb{Q}$ (the standard absolute value). For a nonarchimedean class $v \in M_{k} \backslash M_{k}^{\infty}$, we let $|p|_{v}=p^{-1}$ if $v$ lies above the rational prime $p$. Let \|\|$_{v}=||_{v}^{d_{v} / \mathfrak{d}}$, where $k_{v}$ is the completion of $k$ with respect to $v$ and $d_{v}=\left[k_{v}: \mathbb{Q}_{v}\right]$ is the local degree. Then the following product formula holds for every $x \in k^{*}$ :

$$
\prod_{v \in M_{k}}\|x\|_{v}=1 .
$$

When $L$ is the function field of $V$, where $V \subset \mathbb{P}^{M_{0}}$ is a nonsingular projective variety over an algebraically closed field of characteristic 0 , we let $M_{L}$ be the set of prime divisors of $V$ (irreducible subvarieties of codimension one). Let $\mathcal{P} \in M_{L}$ be such a prime divisor. As $V$ is nonsingular, the local ring $\mathcal{O}_{\mathcal{P}}$ at $\mathcal{P}$ is a discrete valuation ring. For each $x \in L^{*}$, its order $\operatorname{ord}_{\mathcal{P}} x$ at $\mathcal{P}$ is well defined. We can associate to $x$ its divisor

$$
(x)=\sum_{\mathcal{P} \in M_{L}} \operatorname{ord}_{\mathcal{P}}(x) \mathcal{P}=(x)_{0}-(x)_{\infty},
$$

where $(x)_{0}$ is the zero divisor of $x$ and $(x)_{\infty}$ is the polar divisor of $x$ respectively. Let $\operatorname{deg} \mathcal{P}$ denote the projective degree of $\mathcal{P}$ in $\mathbb{P}^{M_{0}}$. For $x \in L^{*}$ and $\mathcal{P} \in M_{L}$, we define

$$
\|x\|_{\mathcal{P}}=e^{-\operatorname{ord}_{\mathcal{P}}(x) \operatorname{deg} \mathcal{P}} .
$$


Then the product formula

$$
\prod_{\mathcal{P} \in M_{L}}\|x\|_{\mathcal{P}}=1
$$

holds for all $x \in L^{*}$.

For $Q=\left[x_{0}, \ldots, x_{m}\right] \in \mathbb{P}^{m}(K)$, let $\|Q\|_{v}=\max _{0 \leq i \leq m}\left\|x_{i}\right\|_{v}$. Moreover, define the logarithmic height of $Q$ by

$$
h(Q)=\sum_{v \in M_{K}} \log \|Q\|_{v}
$$

By the product formula, its definition is independent of the choice of the representations.

According to [Lan83, Proposition 2.2], for $K$ and $v \in M_{K}$ as described above, there is a unique extension of $v$ to $\bar{K}_{v}$, where $K_{v}$ is the completion of $K$ with respect to the absolute value $v \in M_{K}$ and $\bar{K}_{v}$ is the algebraic closure of $K_{v}$.

Next, define an $M_{K}$-constant to be a collection of real numbers $\left(c_{v}\right)_{v \in M_{K}}$ such that $c_{v}=0$ for all but finitely many $v$. A collection of functions $\left(\gamma_{v}: X \rightarrow \mathbb{R}\right)_{v \in M_{K}}$ is said to be $O_{M_{K}}(1)$ (or $M_{K}$-bounded) if there exists an $M_{K}$-constant $\left(c_{v}\right)_{v \in M_{K}}$ such that $\left|\gamma_{v}(x)\right| \leq c_{v}$ for all $x \in X$ and $v \in M_{K}$.

Before we define the notion of a global Weil function in Definition 2.5, we first prove the following lemma.

Lemma 2.4. Let $X$ be a projective nonsingular variety and $D$ be an effective divisor on $X$, both defined over $K$. Let $\mathcal{O}_{X}(D)$ be the line bundle associated with $D$, and write $\mathcal{O}_{X}(D)=M_{1} \otimes M_{2}^{-1}$, where $M_{1}, M_{2}$ are very ample line bundles. Choose generating sections $l_{1}, \ldots, l_{t_{1}}$ of $M_{1}$ and $m_{1}, \ldots, m_{t_{2}}$ of $M_{2}$. Let $s_{1}, \ldots, s_{t}$ be arbitrary sections of $\mathcal{O}_{X}(D)$, and $s_{D}$ a section of $\mathcal{O}_{X}(D)$ such that $\operatorname{div}\left(s_{D}\right)=D$. Then, for every $v \in M_{K}$ and $P \notin \operatorname{Supp}(D)$,

$$
\max _{\mu=1, \ldots, t} \log \left\|\frac{s_{\mu}}{s_{D}}(P)\right\|_{v} \leq \max _{1 \leq \alpha \leq t_{1}} \min _{1 \leq \beta \leq t_{2}} \log \left\|\frac{l_{\alpha}}{m_{\beta} s_{D}}(P)\right\|_{v}+c_{v},
$$

where $\left(c_{v}\right)_{v \in M_{K}}$ is an $M_{K}$-constant. Note that we use the notation $m_{\beta} s_{D}$ for $m_{\beta} \otimes s_{D}$ and that $\frac{l_{\alpha}}{m_{\beta} s_{D}}$ is a well-defined rational function on $X$.

Proof. For each $1 \leq \mu \leq t, P \notin \operatorname{Supp}(D)$, and each $1 \leq \alpha_{0} \leq t_{1}$ with $l_{\alpha_{0}}(P) \neq 0$, we take $1 \leq \beta_{0} \leq t_{2}$ such that

$$
\log \left\|\frac{l_{\alpha_{0}}}{m_{\beta_{0}} s_{D}}(P)\right\|_{v}=\min _{1 \leq \beta \leq t_{2}} \log \left\|\frac{l_{\alpha_{0}}}{m_{\beta} s_{D}}(P)\right\|_{v} .
$$

Note that since $l_{1}, \ldots, l_{t_{1}}$, as well $m_{1}, \ldots, m_{t_{2}}$, do not have common zeros, such $\alpha_{0}$ and $\beta_{0}$ exist with the above minimum being finite. Hence

$$
\begin{aligned}
& \log \left\|\frac{s_{\mu}}{s_{D}}(P)\right\|_{v} \\
= & \log \left\|\frac{l_{\alpha_{0}}}{m_{\beta_{0}} s_{D}}(P)\right\|_{v}+\log \left\|\frac{m_{\beta_{0}} s_{\mu}}{l_{\alpha_{0}}}(P)\right\|_{v} \\
= & \min _{1 \leq \beta \leq t_{2}} \log \left\|\frac{l_{\alpha_{0}}}{m_{\beta} s_{D}}(P)\right\|_{v}+\log \left\|\frac{m_{\beta_{0}} s_{\mu}}{l_{\alpha_{0}}}(P)\right\|_{v} \\
\leq & \max _{1 \leq \alpha \leq t_{1}} \min _{1 \leq \beta \leq t_{2}} \log \left\|\frac{l_{\alpha}}{m_{\beta} s_{D}}(P)\right\|_{v}+\max _{\left\{(\alpha, \beta) \mid l_{\alpha}(P) \neq 0\right\}} \log \left\|\frac{m_{\beta} s_{\mu}}{l_{\alpha}}(P)\right\|_{v} .
\end{aligned}
$$


Inequality (4) holds because $\alpha_{0}$ was chosen such that $l_{\alpha_{0}}(P) \neq 0$. To prove the lemma, it now suffices to establish the following claim: for all $v \in M_{K}$,

$$
\max _{1 \leq \mu \leq t\left\{(\alpha, \beta) \mid l_{\alpha}(P) \neq 0\right\}} \log \left\|\frac{m_{\beta} s_{\mu}}{l_{\alpha}}(P)\right\|_{v} \leq c_{v}
$$

for some $M_{K}$-constant $\left(c_{v}\right)_{v \in M_{K}}$.

To prove the claim, we bound by an $M_{K}$-constant, for arbitrary fixed indices $\hat{\beta}, \hat{\mu}, \hat{\alpha}$, the expression

$$
\log \left\|\frac{m_{\hat{\beta}} s_{\hat{\mu}}}{l_{\hat{\alpha}}}(P)\right\|_{v}
$$

for $P \in U_{\hat{\alpha}}=\left\{P \in X \backslash \operatorname{Supp}(D) \mid l_{\hat{\alpha}}(P) \neq 0\right\}$.

To get started, we choose a closed embedding of $X$ into $\mathbb{P}^{N}$ with the standard coordinates $\left[x_{0}, \ldots, x_{N}\right]$. We define, for fixed $0 \leq i \leq N$ and $v \in M_{K}$,

$$
E_{i v}:=\left\{P \in X(\bar{K}) \mid\left\|x_{i}(P)\right\|_{v}=\max _{0 \leq i^{\prime} \leq N}\left\|x_{i^{\prime}}(P)\right\|_{v}\right\} .
$$

Obviously, $\cup_{i=0}^{N} E_{i v}=X$ for each $v \in M_{K}$, so we only need to prove the claim on $E_{i_{0} v} \cap U_{\hat{\alpha}}$ with an arbitrary but fixed $0 \leq i_{0} \leq N$. Without loss of generality, we assume that $i_{0}=0$ and also write $U:=\left\{P \in X(\bar{K}) \mid x_{0}(P) \neq 0\right\}$, noting $E_{0 v} \subset U$.

We define, on $U, f_{i}:=x_{i} / x_{0}$ for $0 \leq i \leq N$. In particular, note that $f_{0} \equiv 1$. Then,

$$
\sup _{P \in E_{0 v}}\left\|f_{i}(P)\right\|_{v} \leq 1
$$

for all $0 \leq i \leq N$, and the regular functions $f_{i}, 0 \leq i \leq N$, generate $K[U]$, i.e., the $K$-algebra of regular functions on $U$.

Next, we define the following divisors on $X$ (with $\left.1 \leq \alpha \leq t_{1}\right): A=\operatorname{div}\left(x_{0}\right)$ and $D_{\alpha}=\operatorname{div}\left(l_{\alpha}\right)$. Since $A$ is ample, it is well-known that, for $m$ a sufficiently large integer, the divisor $m A-D_{\alpha}$ is ample again for all $\alpha=1, \ldots, t_{1}$. Therefore, there is a positive integer $m^{\prime}$ such that $m^{\prime}\left(m A-D_{\alpha}\right)$ is very ample. For every fixed $\alpha$, we pick sections $s_{\alpha j} \in H^{0}\left(X, \mathcal{O}_{X}\left(m^{\prime}\left(m A-D_{\alpha}\right)\right)\right), j=1, \ldots, \operatorname{dim} X+1$, without common zeros. Moreover, let $s_{m^{\prime} m A} \in H^{0}\left(X, \mathcal{O}_{X}\left(m^{\prime} m A\right)\right)$ be such that $\operatorname{div}\left(s_{m^{\prime} m A}\right)=m^{\prime} m A$. Now, for $\alpha=1, \ldots, t_{1}$ and $j=1, \ldots, \operatorname{dim} X+1$, we let

$$
h_{\alpha j}:=\left.\frac{l_{\alpha}^{m^{\prime}} s_{\alpha j}}{s_{m^{\prime} m A}}\right|_{U} .
$$

Each $h_{\alpha j}$ is a regular function on $U$ such that

$$
\tilde{U}_{\alpha}:=\left\{P \in U \mid l_{\alpha} \neq 0\right\} \supseteq\left\{P \in U \mid h_{\alpha j} \neq 0\right\} .
$$

Note that the functions $h_{\alpha j}$ have no common zeros, for the following reason. Since the $l_{\alpha}$ have no common zeros, for every $x \in U$, there is an index $\alpha^{\prime}$ such that $l_{\alpha^{\prime}}(x) \neq 0$. Since the $s_{\alpha^{\prime} j}, j=1, \ldots, \operatorname{dim} X+1$, have no common zeros, there is an index $j^{\prime}$ with $s_{\alpha^{\prime} j^{\prime}}(x) \neq 0$. Hence, $h_{\alpha^{\prime} j^{\prime}}(x) \neq 0$.

We will abuse notation and suppress the index $j$, referring to $h_{\alpha j}$ simply as $h_{\alpha}$, for $\alpha=1, \ldots, t_{3}=t_{1}\left(\operatorname{dim}_{X}+1\right)$. Since these functions, now labeled $h_{\alpha}, \alpha=1, \ldots, t_{3}$, 
have no common zeros on $U$, a version of the Nullstellensatz ([BG06, Lemma 2.2.7]), yields the existence of regular functions $g_{\alpha}$ on $U$ such that

$$
\sum_{\alpha=1}^{t_{3}} g_{\alpha} h_{\alpha}=1 .
$$

Also, we can define, for each $\alpha=1, \ldots, t_{3}$,

$$
\tilde{E}_{\alpha v}=\left\{P \in E_{0 v} \mid\left\|h_{\alpha}(P)\right\|_{v}=\max _{1 \leq \alpha^{\prime} \leq t_{1}}\left\|h_{\alpha^{\prime}}(P)\right\|_{v}\right\}
$$

Then, for all $P \in \tilde{E}_{\alpha v}$, for all $1 \leq \alpha^{\prime} \leq t_{3}$,

$$
\left\|\left(h_{\alpha^{\prime}} / h_{\alpha}\right)(P)\right\|_{v} \leq 1 .
$$

Note that $E_{0 v}=\cup_{\alpha=1}^{t_{3}} \tilde{E}_{\alpha v}$. It thus suffices to prove the claim on $\tilde{E}_{\tilde{\alpha} v} \cap U_{\hat{\alpha}}$ for a fixed arbitrary $\tilde{\alpha}$.

Since $f_{i}, 0 \leq i \leq N$, generate the $K$-algebra $K[U]$, there are polynomials $p^{(\alpha)}$ with coefficients $p_{a}^{(\alpha)} \in K$ such that

$$
g_{\alpha}=p^{(\alpha)}\left(f_{0}, \ldots, f_{N}\right)
$$

which implies, due to (6),

$$
\sup _{P \in E_{0 v}}\left\|g_{\alpha}(P)\right\|_{v} \leq C_{\alpha} \max _{a}\left\|p_{a}^{(\alpha)}\right\|_{v}
$$

Concerning the constants $C_{\alpha}$, we remark that when $v$ is one of the finitely many archimedean elements of $M_{K}$, then $C_{\alpha}$ is the number of monomials in $p^{(\alpha)}$. If $v$ is nonarchimedean, then we may take $C_{\alpha}=1$ for $\alpha=1, \ldots, t_{3}$. It is important for our argument to have these constants equal to 1 for almost all $v$.

Next, observe that on $\tilde{E}_{\tilde{\alpha} v}$, we can write, from (7), that

$$
1 / h_{\tilde{\alpha}}=\sum_{\alpha=1}^{t_{3}} g_{\alpha} \frac{h_{\alpha}}{h_{\tilde{\alpha}}} .
$$

Thus, from (10), (8) and (9), we have,

$$
\sup _{P \in \tilde{E}_{\tilde{\alpha} v}}\left\|1 / h_{\tilde{\alpha}}(P)\right\|_{v} \leq \tau \sup _{P \in \tilde{E}_{\tilde{\alpha} v}} \max _{1 \leq \alpha \leq t_{3}}\left\|g_{\alpha}(P)\right\|_{v} \leq \tau \max _{1 \leq \alpha \leq t_{3}}\left(C_{\alpha} \max _{a}\left\|p_{a}^{(\alpha)}\right\|_{v}\right),
$$

where $\tau=1$ for $v$ nonarchimedean, and $\tau=t_{3}$ otherwise.

It follows from [Har77, Proposition II.2.2] that $f_{0}, \ldots, f_{N}$ and $1 / h_{\tilde{\alpha}}$ generate the $K$-algebra $K\left[\tilde{U}_{\tilde{\alpha}}\right]$. We can thus write, on $\tilde{E}_{\tilde{\alpha} v} \cap U_{\hat{\alpha}}$,

$$
\frac{m_{\hat{\beta}} s_{\hat{\mu}}}{l_{\hat{\alpha}}}=\tilde{p}\left(f_{0}, \ldots, f_{N}, 1 / h_{\tilde{\alpha}}\right)
$$

where $\tilde{p}$ is a polynomial with coefficients $\tilde{p}_{a} \in K$. In particular, using (11) and (6), for all $P \in \tilde{E}_{\tilde{\alpha} v} \cap U_{\hat{\alpha}}$,

$$
\begin{aligned}
& \left\|\frac{m_{\hat{\beta}} s_{\hat{\mu}}}{l_{\hat{\alpha}}}(P)\right\|_{v} \\
\leq & \tilde{C} \max _{a}\left\|\tilde{p}_{a}\right\|_{v} \sup _{P \in \tilde{E}_{\tilde{\alpha} v}} \max \left\{\left\|f_{0}(P)\right\|_{v}, \ldots,\left\|f_{N}(P)\right\|_{v},\left\|1 / h_{\tilde{\alpha}}(P)\right\|_{v}\right\} \\
\leq & \left(\tilde{C} \max _{a}\left\|\tilde{p}_{a}\right\|_{v}\right) \cdot\left(\tau \max _{1 \leq \alpha \leq t_{3}} C_{\alpha} \max _{a}\left\|p_{a}^{(\alpha)}\right\|_{v}\right) .
\end{aligned}
$$


Again, the constant $\tilde{C}=1$ for nonarchimedean $v$. Altogether, it is clear now that the expression in the last line is different from 1 only for finitely many $v$. Thus,

$$
\sup _{P \in \tilde{E}_{\tilde{\alpha} v} \cap U_{\hat{\alpha}}} \log \left\|\frac{m_{\hat{\beta}} s_{\hat{\mu}}}{l_{\hat{\alpha}}}(P)\right\|_{v} \leq c_{v},
$$

where $\left(c_{v}\right)_{v \in M_{K}}$ is an $M_{K}$-constant. The claim is proven.

We now define a global Weil function $\left(\lambda_{D, v}\right)_{v \in M_{K}}$ as follows.

DEFinition 2.5. Let $X$ be a projective nonsingular variety and $D$ be an effective divisor on $X$, both defined over $K$. Extend \|\|$_{v}$ to an absolute value on the algebraic closure $\bar{K}_{v}$ for all $v \in M_{K}$. Let $\mathcal{O}_{X}(D)$ be the line bundle associated with $D$. Write $\mathcal{O}_{X}(D)=M_{1} \otimes M_{2}^{-1}$, where $M_{1}, M_{2}$ are very ample line bundles. Now choose generating global sections $l_{1}, \ldots, l_{t_{1}}$ of $M_{1}$ and $m_{1}, \ldots, m_{t_{2}}$ of $M_{2}$. For $P \notin \operatorname{Supp}(D)$, we call the collection of functions

$$
\lambda_{D, v}(P):=\max _{1 \leq \alpha \leq t_{1}} \min _{1 \leq \beta \leq t_{2}} \log \left\|\frac{l_{\alpha}}{m_{\beta} s_{D}}(P)\right\|_{v}
$$

$v \in M_{K}$, a global Weil function, where $s_{D}$ is a section of $\mathcal{O}_{X}(D)$ with $\operatorname{div}\left(s_{D}\right)=D$.

Note that the above definition is indeed independent, up to an $O_{M_{K}}$ (1)-term, of the choice of the auxiliary line bundles and sections made in the definition. In fact, let $\tilde{M}_{1}, \tilde{M}_{2}, \tilde{l}_{1}, \ldots, \tilde{l}_{\tau_{1}}$ and $\tilde{m}_{1}, \ldots, \tilde{m}_{\tau_{2}}$ be another choice of line bundles and bases as in Definition 2.5. Using the same arguments used to arrive at inequality (4) in Lemma 2.4, we have

$$
\begin{aligned}
\max _{1 \leq \delta \leq \tau_{1}} \min _{1 \leq \varepsilon \leq \tau_{2}} \log \left\|\frac{\tilde{l}_{\delta}}{\tilde{m}_{\varepsilon} s_{D}}(P)\right\|_{v} \leq & \max _{1 \leq \alpha \leq t_{1}} \min _{1 \leq \beta \leq t_{2}} \log \left\|\frac{l_{\alpha}}{m_{\beta} s_{D}}(P)\right\|_{v} \\
& +\max _{\left\{(\alpha, \beta, \delta, \varepsilon) \mid\left(\tilde{m}_{\varepsilon} l_{\alpha}\right)(P) \neq 0\right\}} \log \left\|\frac{\tilde{l}_{\delta} m_{\beta}}{\tilde{m}_{\varepsilon} l_{\alpha}}(P)\right\|_{v} .
\end{aligned}
$$

An argument similar to the one used to prove inequality (12) shows that

$$
\max _{\left\{(\alpha, \beta, \delta, \varepsilon) \mid\left(\tilde{m}_{\varepsilon} l_{\alpha}\right)(P) \neq 0\right\}} \log \left\|\frac{\tilde{l}_{\delta} m_{\beta}}{\tilde{m}_{\varepsilon} l_{\alpha}}(P)\right\|_{v} \leq c_{v},
$$

where $\left(c_{v}\right)_{v \in M_{K}}$ is an $M_{K}$-constant. Hence

$$
\max _{1 \leq \delta \leq \tau_{1}} \min _{1 \leq \varepsilon \leq \tau_{2}} \log \left\|\frac{\tilde{l}_{\delta}}{\tilde{m}_{\varepsilon} s_{D}}(P)\right\|_{v} \leq \max _{1 \leq \alpha \leq t_{1}} \min _{1 \leq \beta \leq t_{2}} \log \left\|\frac{l_{\alpha}}{m_{\beta} s_{D}}(P)\right\|_{v}+c_{v} .
$$

The other direction can be obtained in a similar way.

Due to the well-definedness up to $M_{K}$-boundedness, we will sometimes abuse notation in the sequel and write $\lambda_{D, v}=\lambda_{D, v}+c_{v}$ for an $M_{K}$-constant $\left(c_{v}\right)_{v \in M_{K}}$.

Definition 2.6. For a finite set $S \subset M_{K}$ containing all archimedean elements, define the counting function $N_{S}(P, D)$ by

$$
N_{S}(P, D)=\sum_{v \notin S} \lambda_{D, v}(P)
$$

We remark that the sum defining $N_{S}(P, D)$ is always a finite sum for $P \in X(\bar{K})$. 
Next, we define the notion of integral points appropriate for our setting. For a more detailed discussion, including some motivational comments, of this notion, we refer the reader to [Voj87, Chapter 1, §4].

Definition 2.7. A subset $\Sigma$ of $X(\bar{K}) \backslash \operatorname{Supp}(D)$ is $(D, S)$-integral if there is an $M_{K}$-constant $\left(c_{v}\right)_{v \in M_{K}}$ such that for all $v \in M_{K} \backslash S$ and all embeddings $\bar{K} \rightarrow \bar{K}_{v}$,

$$
\lambda_{D, v}(P) \leq c_{v}
$$

for all $P \in \Sigma$. We define the $(D, S)$-integral set $\Sigma$ to be $K$-rational if $\Sigma \subset X(K) \backslash$ $\operatorname{Supp}(D)$. In particular, a subset $\Sigma$ of $X(K) \backslash \operatorname{Supp}(D)$ is $(D, S)$-integral if and only if $N_{S}(\cdot, D)$ is bounded over $\Sigma$.

We recall the following generalized version of Schmidt's Subspace Theorem over number fields from [Voj89].

THEOREM 2.8. Let $k$ be a number field with its set of canonical places $M_{k}$. Let $S \subset M_{k}$ be a finite set containing all archimedean places. Let $H_{1}, \ldots, H_{m}$ be hyperplanes in $\mathbb{P}^{n}$ defined over $\bar{k}$ with corresponding Weil functions $\lambda_{H_{1}}, \ldots, \lambda_{H_{m}}$. Then there exists a finite union of hyperplanes $Z$, depending only on $H_{1}, \ldots, H_{m}$ (and not $k, S$ ), such that for any $\varepsilon>0$,

$$
\sum_{v \in S} \max _{I} \sum_{i \in I} \lambda_{H_{i}, v}(P) \leq(n+1+\varepsilon) h(P)
$$

holds for all but finitely many $P \in \mathbb{P}^{n}(k) \backslash Z$, where the maximum is taken over subsets $I \subset\{1, \ldots, m\}$ such that the linear forms defining $H_{i}, i \in I$, are linearly independent.

For the function field case with $L$ as above, we recall the following effective version of Schmidt's Subspace Theorem from [Wan04].

Theorem 2.9. Let $H_{1}, \ldots, H_{q}$ be hyperplanes in $\mathbb{P}^{n}$ defined over $L$ and $S$ be a finite set of prime divisors of $V$. Then there exists an effectively computable finite union $\mathcal{R}$ of proper linear subspaces of $\mathbb{P}^{n}$, defined over $L$ and depending only on the given hyperplanes, such that the following is true. Given $\epsilon>0$, there exist effectively computable constants $C_{\epsilon}$ and $C_{\epsilon}^{\prime}$ such that for any $\mathrm{x} \in \mathbb{P}^{n}(L) \backslash \mathcal{R}$,

$$
h(\mathbf{x}) \leq C_{\epsilon}
$$

or

$$
\sum_{\mathcal{P} \in S} \max _{J} \sum_{j \in J} \lambda_{H_{j}, \mathcal{P}}(\mathbf{x}) \leq(n+1+\epsilon) h(\mathbf{x})+C_{\epsilon}^{\prime},
$$

where the maximum is taken over all subsets $J$ of $\{1, \ldots, q\}$ such that the linear forms $H_{j}, j \in J$, are linearly independent.

Our main result in this subsection is the following inequality of Schmidt Subspace Theorem-type for essentially large divisors. Recall that the constant $M$ was already defined in Theorem 2.2 .

THEOREM 2.10. Let $D$ be an essentially very large divisor on a nonsingular projective variety $X$, both defined over $K$. Let $V$ be a linear subspace of $L(D)$ as in Definition 1.5. Let $\phi_{0}, \ldots \phi_{m}$ be an arbitrary basis for $V$ and let $\Phi$ be the rational map $\left[\phi_{0}: \ldots: \phi_{m}\right]: X \rightarrow \mathbb{P}^{m}$. 
(a) If $K=k$ is a number field, and $S \subset M_{K}$ be a finite set of elements containing all archimedean ones, then, for every $\varepsilon>0$,

$$
h(\Phi(P)) . \leq .(M(m+1)+1+\varepsilon) N_{S}(P, D),
$$

where ". $\leq$. " means that the inequality holds for all $P \in X(k)$ outside of a Zariski closed subset $Z$ of $X$.

(b) If $K=L$ is the function field of a nonsingular projective variety $V$ over an algebraically closed field of characteristic 0 , and $S$ is a finite set of prime divisors of $V$, then there exists an effectively computable finite union $\mathcal{R}$ of proper subvarieties of $X$ depending only on $\Phi$ such that the following is true. Given $\epsilon>0$, there exist effectively computable constants $C_{\epsilon}$ and $C_{\epsilon}^{\prime}$ such that for any $\mathrm{x} \in X(L) \backslash \mathcal{R}$,

$$
h(\Phi(\mathbf{x})) \leq C_{\epsilon}
$$

or

$$
h(\Phi(\mathbf{x})) \leq(M(m+1)+1+\varepsilon) N_{S}(\mathbf{x}, D)+C_{\epsilon}^{\prime}
$$

Proof. We only prove (a) since the proof of (b) is virtually identical. Due to arguments analogous to those that gave the boundedness (3), we have for $P \in X(k)$ not contained in the support of $D$ :

$$
\sum_{v \in S} \max _{j} \log \left\|\phi_{j}(P)\right\|_{v}^{m+1+\frac{1}{M}} \leq \sum_{v \in S} \max _{I} \log \prod_{L \in I} \frac{\max _{j}\left\|\phi_{j}(P)\right\|_{v}}{\|L(\Phi(P))\|_{v}}+c_{v}
$$

where $\left(c_{v}\right)_{v \in S}$ are finitely many constants. By Schmidt's Subspace Theorem, for all $P \in X(k)$ outside a Zariski closed subset $Z$ of $X$,

$$
\sum_{v \in S} \max _{I} \log \prod_{L \in I} \frac{\max _{j}\left\|\phi_{j}(P)\right\|_{v}}{\|L(\Phi(P))\|_{v}} . \leq .(m+1+\varepsilon) h(\Phi(P)) .
$$

Hence

$$
\sum_{v \in S} \max _{j} \log \left\|\phi_{j}(P)\right\|_{v}^{m+1+\frac{1}{M}} \cdot \leq .(m+1+\varepsilon) h(\Phi(P))+\sum_{v \in S} c_{v} .
$$

We remark that the constant $\sum_{v \in S} c_{v}$ can be absorbed into the term $(m+1+$ $\varepsilon) h(\Phi(P))$ and thus be dropped from the inequality. Moreover, by definition,

$$
\sum_{v \in S} \max _{j} \log \left\|\phi_{j}(P)\right\|_{v}+\sum_{v \notin S} \max _{j} \log \left\|\phi_{j}(P)\right\|_{v}=h(\Phi(P)) .
$$

We again use the fact that, due to the isomorphism (1), there are sections $s_{0}, \ldots, s_{m} \in H^{0}\left(X, \mathcal{O}_{X}(D)\right)$ such that $\phi_{j}=\frac{s_{j}}{s_{D}}, j=0, \ldots, m$, where $s_{D}$ is a section of $\mathcal{O}_{X}(D)$ with $\operatorname{div}\left(s_{D}\right)=D$. Thus

$$
\sum_{v \in S} \max _{j} \log \left\|\phi_{j}(P)\right\|_{v}+\sum_{v \notin S} \max _{j} \log \left\|\frac{s_{j}}{s_{D}}(P)\right\|_{v}=h(\Phi(P)) .
$$


From Lemma 2.4,

$$
\max _{j} \log \left\|\frac{s_{j}}{s_{D}}(P)\right\|_{v} \leq \lambda_{D, v}
$$

Therefore,

$$
\sum_{v \in S} \max _{j} \log \left\|\phi_{j}(P)\right\|_{v}+\sum_{v \notin S} \lambda_{D, v}(P) \geq h(\Phi(P)) .
$$

So we have

$$
\begin{gathered}
\left(m+1+\frac{1}{M}\right) h(\Phi(P)) \\
\leq \sum_{v \in S} \max _{j} \log \left\|\phi_{j}(P)\right\|_{v}^{m+1+\frac{1}{M}}+\left(m+1+\frac{1}{M}\right) \sum_{v \notin S} \lambda_{D, v}(P) \\
. \leq .(m+1+\varepsilon) h(\Phi(P))+\left(m+1+\frac{1}{M}\right) N_{S}(P, D) .
\end{gathered}
$$

After rearranging the inequality and choosing $\varepsilon$ and $\delta$ (depending on $\varepsilon$ ) small, we obtain the Theorem.

In strict analogy to Corollary 2.3, we obtain the following

COROLlARY 2.11. Let $D$ be an essentially large divisor on a nonsingular projective variety $X$, both defined over a number field $k$. Let $S \subset M_{k}$ be a finite set of places containing all archimedean ones. Then any set of $\hat{k}$-rational $(D, S)$-integral points is contained in a proper subvariety of $X$ for any finite extension $\hat{k}$ of $k$.

Proof. We can assume without loss of generality that $\hat{k}=k$ and $D$ is essentially very large. Let $\Sigma$ be a set of $(D, S)$-integral points. According to Definition 2.7, $N_{S}(\cdot, D)$ is bounded over $\Sigma$. So, by Theorem $2.10($ a $), h(\Phi(P))$ is bounded for all $P \in \Sigma$ outside a Zariski closed subset $Z$ of $X$. This means that, due to Northcott's Theorem, the set $\{\Phi(P) \mid P \in(\Sigma \backslash Z)(k)\}$ is finite. Since $\Phi$ is a nonconstant morphism outside of $D$, the preimage of this finite set is a proper Zariski closed subset $Z^{\prime}$ of $X$. We have shown that $\Sigma \subset Z \cup Z^{\prime}$, which concludes the proof.

3. Proofs of the sharp sufficient criteria for essentially large divisors. As was stated in the Introduction, the filtration method from [CZ04a], [Ru04] can be used to prove the sharp bound for the number of components of a large divisor in the case of $X=\mathbb{P}^{q}$ (Theorem 1.7). We now give the proof. In doing so, we return to the general setting where we are working over an arbitrary field $K$ of characteristic zero.

Proof of Theorem 1.7. Let $Q_{1}, \ldots, Q_{r}$ be homogeneous polynomials of degree $d_{i}$ such that $D_{i}=\left\{Q_{i}=0\right\}$. Let $P \in D(\bar{K})$. After replacing each $Q_{i}$ by $Q_{i}^{d / d_{i}}$, where $d$ is the least common multiple of the $d_{i}$ 's, we can assume that all $Q_{i}$ have the same degree $d$. Without loss of generality, assume that precisely $q$ of the $D_{i}(\bar{K})$ contain the point $P$, and let $\left\{\gamma_{1}, \ldots, \gamma_{q}\right\}$ be the unique set of distinct elements of $\left\{Q_{1}, \ldots, Q_{r}\right\}$ such that $P \in \cap_{i=1}^{q}\left\{P \in X(\bar{K}) \mid \gamma_{i}(P)=0\right\}$.

Let $V_{N}$ be the vector space of homogeneous polynomials of degree $N$ in $K\left[X_{0}, \ldots, X_{q}\right]$. With the lexicographical ordering on the $q$-tuples $\vec{i}=\left(i_{1}, \ldots, i_{q}\right)$ and $\sigma(\vec{i}):=\sum_{j=1}^{q} i_{j} \leq N / d$, we obtain a filtration on $V_{N}$ by letting

$$
W_{\vec{i}}=\sum_{\vec{e} \geq \vec{i}} \gamma_{1}^{e_{1}} \ldots \gamma_{q}^{e_{q}} V_{N-d \sigma(\vec{e})}
$$


Note that clearly $W_{\overrightarrow{0}}=V_{N}$ and $W_{\vec{i}} \supseteq W_{\vec{i}^{\prime}}$ for $\overrightarrow{i^{\prime}} \geq \vec{i}$. Combining Lemma 2.3 and Lemma 3.1 of [CZ04a], we note the following lemma for our situation.

Lemma 3.1. There exists an integer $N_{0}$ (depending only on $\gamma_{1}, \ldots, \gamma_{q}$ ) such that for all integers $N>N_{0}$ and for all $\vec{i}$ with $d \sigma(\vec{i})<N-N_{0}$ and immediate successor $\overrightarrow{i^{\prime}}$

$$
\Delta_{\vec{i}}:=\operatorname{dim} W_{\vec{i}} / W_{\vec{i}^{\prime}}=d^{q} .
$$

Now choose a basis $\psi_{1}, \ldots, \psi_{m}\left(m=\left(\begin{array}{c}N+q \\ q\end{array}\right)\right)$ for $V_{N}$ with respect to the above filtration. For all $\nu=1, \ldots, m$, write

$$
\psi_{\nu}=\gamma_{1}^{i_{1}} \ldots \gamma_{q}^{i_{q}} \gamma^{(\nu)}
$$

for some $\gamma^{(\nu)} \in V_{N-d \sigma(\vec{i})}$ according to its place in the filtration.

For $\tilde{N} \in \mathbb{N}$, let $N=\tilde{N} \cdot r \cdot d$ and write

$$
H^{0}\left(\mathbb{P}^{q}, \mathcal{O}_{\mathbb{P}^{q}}(\tilde{N} D)\right)=H^{0}\left(\mathbb{P}^{q}, \mathcal{O}_{\mathbb{P}^{q}}(\tilde{N} \cdot r \cdot d)\right)=H^{0}\left(\mathbb{P}^{q}, \mathcal{O}_{\mathbb{P}^{q}}(N)\right)=V_{N}
$$

For $\nu=1, \ldots, m$, let

$$
f_{\nu}=\frac{\psi_{\nu}}{Q_{1}^{\tilde{N}} \ldots Q_{r}^{\tilde{N}}}
$$

These rational functions clearly form a basis for $L(\tilde{N} D)$. To conclude the proof of the theorem, we show that they satisfy $\operatorname{ord}_{E} \prod_{\nu=1}^{m} f_{\nu}>0$ for any component $E$ in $D$ with $P \in E(\bar{K})$. We assume that $E$ is contained in $D_{j_{0}}$. Then

$$
\frac{1}{\operatorname{ord}_{E} D} \operatorname{ord}_{E} \prod_{\nu=1}^{m} f_{\nu}=\left(\sum_{\vec{i}} \Delta_{\vec{i}} i_{j_{0}}\right)-\tilde{N}\left(\begin{array}{c}
N+q \\
q
\end{array}\right)
$$

Since the number of nonnegative integer $m$-tuples with sum $\leq t$ is equal to the number of nonnegative integer $(m+1)$-tuples with sum exactly $t$, which is $\left(\begin{array}{c}t+m \\ m\end{array}\right)$, and since the sum below is independent of $j$, we have that, for $N$ divisible by $d$ and for every $j$,

$$
\begin{aligned}
\sum_{\vec{i}} i_{j} & =\frac{1}{q+1} \sum_{\hat{\vec{i}}} \sum_{\eta=1}^{q+1} i_{\eta}=\frac{1}{q+1} \sum_{\hat{\vec{i}}} \frac{N}{d} \\
& =\frac{1}{q+1}\left(\begin{array}{c}
N / d+q \\
q
\end{array}\right) \frac{N}{d}=\frac{N^{q+1}}{d^{q+1}(q+1) !}+O\left(N^{q}\right)
\end{aligned}
$$

where $\sum_{\hat{\vec{i}}}$ is taken over all nonnegative integer $(q+1)$-tuples with sum exactly $N / d$.

Combining it with Lemma 3.1, we have, for every $1 \leq j_{0} \leq q$,

$$
\sum_{\vec{i}} \Delta_{\vec{i}} i_{j_{0}}=\frac{N^{q+1}}{d(q+1) !}+O\left(N^{q}\right)
$$


Thus,

$$
\begin{aligned}
\frac{1}{\operatorname{ord}_{E} D} \operatorname{ord}_{E} \prod_{\nu=1}^{m} f_{\nu} & =\left(\sum_{\vec{i}} \Delta_{\vec{i}} i_{j_{0}}\right)-\tilde{N}\left(\begin{array}{c}
N+q \\
q
\end{array}\right) \\
& =\left(\frac{N^{q+1}}{d \cdot(q+1) !}+O\left(N^{q}\right)\right)-\frac{N}{d r}\left(\frac{N^{q}}{q !}+O\left(N^{q-1}\right)\right) \\
& =\left(\frac{1}{q+1}-\frac{1}{r}\right) \frac{N^{q+1}}{d \cdot q !}+O\left(N^{q}\right) .
\end{aligned}
$$

For $N$ sufficiently large, this is positive if $r \geq q+2$.

We continue with the proof of Theorem 1.8, based on the methods of [Fer00], [EF02], [EF08] and [Ru09].

Proof of Theorem 1.8. Let $Q_{1}, \ldots, Q_{r}$ be homogeneous polynomials such that $D_{i}=\operatorname{div}\left(\left.Q_{i}\right|_{X}\right)$. As above, we can assume that the degrees of the homogeneous polynomials $Q_{i}$ equal $d$ for all $i$. Let

$$
\varphi: X \rightarrow \mathbb{P}^{r-1}, \quad x \mapsto\left[Q_{1}(x), \ldots, Q_{r}(x)\right] .
$$

Let $Y:=\varphi(X)$. By the general position assumption, $\varphi$ is a finite morphism $X \rightarrow Y$.

On $\mathbb{P}^{r-1}$, we have for all $N \in \mathbb{N}$ a short exact sequence

$$
0 \rightarrow \mathcal{I}_{Y}(N) \rightarrow \mathcal{O}_{\mathbb{P}^{r-1}}(N) \rightarrow \mathcal{O}_{Y}(N) \rightarrow 0 .
$$

The beginning of the corresponding long exact sequence reads

$$
0 \rightarrow H^{0}\left(\mathbb{P}^{r-1}, \mathcal{I}_{Y}(N)\right) \rightarrow H^{0}\left(\mathbb{P}^{r-1}, \mathcal{O}_{\mathbb{P}^{r-1}}(N)\right) \stackrel{\tau}{\rightarrow} H^{0}\left(Y, \mathcal{O}_{Y}(N)\right),
$$

where $\tau$ denotes the restriction map. For $N$ a positive multiple of $r$, we let

$$
\begin{aligned}
& W_{N}:=\operatorname{image}(\tau) \\
& \cong H^{0}\left(\mathbb{P}^{r-1}, \mathcal{O}_{\mathbb{P}^{r-1}}(N)\right) / \operatorname{ker}(\tau) \\
& \cong H^{0}\left(\mathbb{P}^{r-1}, \mathcal{O}_{\mathbb{P}^{r-1}}(N)\right) / H^{0}\left(\mathbb{P}^{r-1}, \mathcal{I}_{Y}(N)\right) \\
& \cong K\left[Y_{0}, \ldots, Y_{r-1}\right]_{N} /\left(I_{Y}\right)_{N},
\end{aligned}
$$

where $\left(I_{Y}\right)_{N}$ denotes the set of those homogeneous polynomials of degree $N$ vanishing on $Y$. Let $V_{N}:=\varphi^{*}\left(W_{N}\right) \subseteq H^{0}\left(X, \mathcal{O}_{X}\left(\frac{N}{r} D\right)\right)$.

Since $\varphi: X \rightarrow Y$ is a finite surjective morphism,

$$
\operatorname{dim}\left(V_{N}\right)=\operatorname{dim}\left(W_{N}\right)=\operatorname{dim} K\left[Y_{0}, \ldots, Y_{r-1}\right]_{N} /\left(I_{Y}\right)_{N}=H_{Y}(N),
$$

where $H_{Y}(N)$ is the Hilbert function of $Y$.

Let $P \in D(\bar{K})$. Without loss of generality, we assume again that there are distinct $Q_{i_{1}}, \ldots, Q_{i_{q}} \in\left\{Q_{1}, \ldots, Q_{r}\right\}$ such that $P \in \cap_{j=1}^{q}\left\{P \in X(\bar{K}) \mid Q_{i_{j}}(P)=0\right\}$.

Let $\vec{c}=\left(c_{1}, \ldots, c_{r}\right)$ be the $r$-vector whose $i_{j}$-th entry $(1 \leq j \leq q)$ is 1 , with all other entries being 0 . Let $\vec{y}^{(1)}, \ldots, \vec{y}^{\vec{a}^{\left(H_{Y}(N)\right)}}$ be monomials such that their equivalence classes in $K\left[Y_{0}, \ldots, Y_{r-1}\right]_{N} /\left(I_{Y}\right)_{N}$ give a basis and such that

$$
S_{Y}(N, \vec{c})=\sum_{i=1}^{H_{Y}(N)} \vec{a}^{(i)} \bullet \vec{c}
$$


where $S_{Y}(N, \vec{c})$ is the $N$-th Hilbert weight and the bullet denotes the usual dot product. Recall that the $N$-th Hilbert weight is given by

$$
S_{Y}(N, \vec{c})=\max \sum_{i=1}^{H_{Y}(N)} \vec{a}^{(i)} \bullet \vec{c}
$$

where the maximum is taken over all sets of monomials $\vec{y}^{(1)}, \ldots, \vec{y}^{\left(H_{Y}(N)\right)}$ whose residue class modulo $I_{Y}$ form a basis of $K\left[Y_{0}, \ldots, Y_{r-1}\right]_{N} /\left(I_{Y}\right)_{N}$. For $\nu=$ $1, \ldots, H_{Y}(N)$, and $N$ a positive multiple of $r$, let

$$
f_{\nu}=\left.\frac{Q_{1}^{a_{1}^{(\nu)}} \ldots Q_{r}^{a_{r}^{(\nu)}}}{Q_{1}^{N / r} \ldots Q_{r}^{N / r}}\right|_{X}
$$

These functions form a basis for $V_{N}$ understood as a subspace of $L\left(\frac{N}{r} D\right)$. To conclude the proof of the theorem, we show that they satisfy $\operatorname{ord}_{E} \prod_{\nu=1}^{H_{Y}(N)} f_{\nu}>0$ for any component $E$ in $D$ with $P \in E(\bar{K})$. We assume that $E$ is contained in $D_{j_{0}}$.

We recall two basic lemmas from [EF02], [EF08], and [Ru09]. These references cover the cases of $\mathbb{C}$ and number fields. The proof in the function field case is completely analogous.

LEMma 3.2. Let $X \subset \mathbb{P}^{N}$ be an algebraic variety of dimension $n$ and degree $\triangle$. Let $m>\triangle$ be an integer and let $\vec{c}=\left(c_{0}, \ldots, c_{N}\right) \in \mathbb{R}_{\geq 0}^{N+1}$. Then

$$
\frac{1}{m H_{X}(m)} S_{X}(m, \vec{c}) \geq \frac{1}{(n+1) \triangle} e_{X}(\vec{c})-\frac{(2 n+1) \triangle}{m} \cdot\left(\max _{i=0, \ldots, N} c_{i}\right),
$$

where $\frac{1}{(n+1) \triangle} e_{X}(\vec{c})$ is the normalized Chow weight of $X$ with respect to $\vec{c}$.

We will not give a definition of the normalized Chow weight here and instead refer the reader to the above references. For our purposes, all we need is the estimate from below contained in the next lemma. We remark that the use of the Chow weight in this context was first introduced by Ferretti [Fer00], who was able to relate it to the probability measure introduced by Faltings-Wüstholz [FW94] in their proof of arithmetic inequalities of Subspace Theorem-type based on Faltings' Product Theorem.

Lemma 3.3. Let $Y$ be a subvariety of $\mathbb{P}^{q-1}$ of dimension $n$ and degree $\triangle$. Let $\vec{c}=$ $\left(c_{1}, \ldots, c_{q}\right)$ be a tuple of non-negative reals. Let $\left\{i_{0}, \ldots, i_{n}\right\}$ be a subset of $\{1, \ldots, q\}$ such that

$$
Y(\bar{K}) \cap\left\{y_{i_{0}}=0, \ldots, y_{i_{n}}=0\right\}=\emptyset
$$

Then

$$
e_{Y}(\vec{c}) \geq\left(c_{i_{0}}+\ldots+c_{i_{n}}\right) \cdot \triangle
$$

We now continue our proof. With our chosen $\vec{c}$ and $\vec{a}^{(i)}$, using Lemmas 3.2 and 3.3 (notice the condition that $Q_{1}, \ldots, Q_{r}$ are in general position), and the symmetry 
property of the $\vec{a}^{(1)}, \ldots, \vec{a}^{\left(H_{Y}(N)\right)}([\mathrm{Mum} 77$, p. 61]),

$$
\begin{aligned}
\frac{1}{\operatorname{ord}_{E} D} \operatorname{ord}_{E} \prod_{\nu=1}^{H_{Y}(N)} f_{\nu} & =\left(\sum_{\nu=1}^{H_{Y}(N)} a_{j_{0}}^{(\nu)}\right)-\frac{N}{r} H_{Y}(N) \\
& =\frac{1}{q}\left(\sum_{\nu=1}^{H_{Y}(N)} \vec{a}^{(\nu)} \bullet \vec{c}\right)-\frac{N}{r} H_{Y}(N) \\
& =\frac{1}{q} S_{Y}(N, \vec{c})-\frac{N}{r} H_{Y}(N) \\
& \geq \frac{1}{q} \frac{1}{q+1} N H_{Y}(N)\left(\sum_{j=1}^{q} c_{i_{j}}\right)-O\left(H_{Y}(N)\right)-\frac{N}{r} H_{Y}(N) \\
& =\frac{1}{q+1} N H_{Y}(N)-\frac{N}{r} H_{Y}(N)-O\left(H_{Y}(N)\right) \\
& =\left(\frac{1}{q+1}-\frac{1}{r}\right) N H_{Y}(N)-O\left(H_{Y}(N)\right) .
\end{aligned}
$$

For $N$ sufficiently large, this is positive if $r \geq q+2$.

4. Essentially large divisors on projective varieties with $\mathrm{Pic}=\mathbb{Z}$. Our ultimate (and so far unreached) goal is of course to find a (sharp) lower bound for the number of (say, big) components in general position necessary for a divisor on a nonsingular projective variety to be essentially large (cf. Conjecture 1.10). For a general nonsingular projective variety, it is far from true that (multiples of) effective big divisors are cut out by hypersurfaces in the ambient projective space, as assumed in Theorem 1.8. In this final section, we prove, basically for lack of a reference, that for a nonsingular projective variety with $\mathrm{Pic}=\mathbb{Z}$, up to taking multiples, effective divisors are cut out by hypersurfaces. Thus, Theorem 1.8 can be applied. Consequently, the above-mentioned ultimate goal has been achieved in the case of $\mathrm{Pic}=\mathbb{Z}$, as formulated in the following corollary. We continue to work over an arbitrary field $K$ of characteristic zero.

Corollary 4.1. Let $q \geq 1$ and $r \geq q+2$ be integers. Let $X \subseteq \mathbb{P}^{\ell}$ be a nonsingular projective variety of dimension $q$, defined over $K$. Assume that $\operatorname{Pic}(X)=\mathbb{Z}$. Let $D=\sum_{i=1}^{r} D_{i}$ be an effective divisor on $X$ defined over $K$ such that the $D_{i}$ are in general position. Then $D$ is essentially large.

Proof. Due to Theorem 1.8, the corollary is proven once we have established that there exist positive integers multiples of each $D_{i}$ that are defined by the restriction to $X$ of a homogeneous polynomial in $K\left[X_{0}, \ldots, X_{\ell}\right]$.

To this end, consider the line bundle $\mathcal{O}_{X}\left(D_{i}\right)$, which represents an element in $\operatorname{Pic}(X)$. Due to $\operatorname{Pic}(X)=\mathbb{Z}$, there exist positive integers $m_{i}, \kappa_{i}$ such that $\mathcal{O}_{X}\left(m_{i} D_{i}\right)=\mathcal{O}_{X}\left(\kappa_{i}\right)$ as elements of $\operatorname{Pic}(X)$. We are free to multiply this equality with an arbitrary positive integer $\tilde{m}_{i}$, obtaining $\mathcal{O}_{X}\left(\tilde{m}_{i} m_{i} D_{i}\right)=\mathcal{O}_{X}\left(\tilde{m}_{i} \kappa_{i}\right)$.

Consider the short exact sequence

$$
0 \rightarrow \mathcal{I}_{X}\left(\tilde{m}_{i} \kappa_{i}\right) \rightarrow \mathcal{O}_{\mathbb{P}^{\ell}}\left(\tilde{m}_{i} \kappa_{i}\right) \rightarrow \mathcal{O}_{X}\left(\tilde{m}_{i} \kappa_{i}\right) \rightarrow 0
$$


The corresponding long exact sequence starts with

$$
\begin{aligned}
0 & \rightarrow H^{0}\left(\mathbb{P}^{\ell}, \mathcal{I}_{X}\left(\tilde{m}_{i} \kappa_{i}\right)\right) \rightarrow H^{0}\left(\mathbb{P}^{\ell}, \mathcal{O}_{\mathbb{P}^{\ell}}\left(\tilde{m}_{i} \kappa_{i}\right)\right) \rightarrow H^{0}\left(X, \mathcal{O}_{X}\left(\tilde{m}_{i} \kappa_{i}\right)\right) \\
& \rightarrow H^{1}\left(\mathbb{P}^{\ell}, \mathcal{I}_{X}\left(\tilde{m}_{i} \kappa_{i}\right)\right)
\end{aligned}
$$

Due to Serre's Vanishing Theorem, $H^{1}\left(\mathbb{P}^{\ell}, \mathcal{I}_{X}\left(\tilde{m}_{i} \kappa_{i}\right)\right)=0$ when $\tilde{m}_{i}$ is sufficiently large. Thus, all sections in $H^{0}\left(X, \mathcal{O}_{X}\left(\tilde{m}_{i} \kappa_{i}\right)\right)$ extend to sections in $H^{0}\left(\mathbb{P}^{\ell}, \mathcal{O}_{\mathbb{P} \ell}\left(\tilde{m}_{i} \kappa_{i}\right)\right)$ when $\tilde{m}_{i}$ is sufficiently large. In particular, sections $s_{i} \in H^{0}\left(X, \mathcal{O}_{X}\left(\tilde{m}_{i} \kappa_{i}\right)\right)$ with

$$
\operatorname{div}\left(s_{i}\right)=\tilde{m}_{i} m_{i} D_{i}
$$

extend. However, the sections of $\mathcal{O}_{\mathbb{P} \ell}\left(\tilde{m}_{i} \kappa_{i}\right)$ correspond to homogeneous polynomials of degree $\tilde{m}_{i} \kappa_{i}$ in $K\left[X_{0}, \ldots, X_{\ell}\right]$. Thus, $\tilde{m}_{i} m_{i} D_{i}$ is indeed defined by the restriction to $X$ of a homogeneous polynomial in $K\left[X_{0}, \ldots, X_{\ell}\right]$ when $\tilde{m}_{i}$ is sufficiently large.

Finally, we exhibit a large class of nonsingular projective varieties that satisfy the Pic $=\mathbb{Z}$ assumption in Corollary 4.1, namely those of sufficiently small codimension . We refer the reader to [Har74] or [Laz04, Section 3.2] for more on the algebraic geometry behind this question, which is essentially the problem of extending the Lefschetz Hyperplane Theorem to nonsingular varieties that are not complete intersections. In particular, the theorems of Barth [Bar70], Larsen [Lar73], and, in the case of a general ground field of characteristic zero, Ogus [Ogu73] apply. For our purposes, we simply state the following proposition, which is an immediate consequence of these theorems.

Proposition 4.2. Let $X \subset \mathbb{P}^{\ell}$ be a nonsingular projective variety of dimension $q$, defined over $K$. If $2 q-\ell \geq 2$, then restriction yields an isomorphism

$$
\operatorname{Pic}\left(\mathbb{P}^{\ell}\right) \stackrel{\cong}{\rightarrow} \operatorname{Pic}(X) \text {. }
$$

Since $\operatorname{Pic}\left(\mathbb{P}^{\ell}\right)=\mathbb{Z}$, the proposition gives the following immediate consequence of Corollary 4.1 .

Corollary 4.3. Let $q \geq 1$ and $r \geq q+2$ be integers. Let $X \subseteq \mathbb{P}^{\ell}$ be a nonsingular projective variety of dimension $q$, defined over $K$. Assume that $2 q-\ell \geq 2$ holds. Let $D=\sum_{i=1}^{r} D_{i}$ be an effective divisor on $X$ defined over $K$ such that the $D_{i}$ are in general position. Then $D$ is essentially large.

We conclude with a comment on the nature of the condition $2 q-\ell \geq 2$. It is well-known that, by general linear projections, any nonsingular projective variety of dimension $q$ can be embedded in $\mathbb{P}^{\ell}$ with $\ell=2 q+1$. Thus, for an arbitrary nonsingular projective variety, one can always find an embedding with $2 q-\ell=-1$. In particular, Corollary 4.3 applies to many interesting special projective varieties such as hypersurfaces, appropriate complete intersections, and certain Grassmannians, such as $G(2,5)$ embedded into $\mathbb{P}^{9}$ under the Plücker embedding. Note that the latter is not a complete intersection due to Bézout's Theorem, because its degree is (the prime number) 5 , while it is not contained in any hyperplane.

\section{REFERENCES}

[Aut09] P. Autissier, Géométries, points entiers et courbes entières, Ann. Sci. Éc. Norm. Supér. (4), 42:2 (2009), pp. 221-239. 
[Bar70] W. BARTh, Transplanting cohomology classes in complex-projective space, Amer. J. Math., 92 (1970), pp. 951-967.

[BG06] E. Bombieri And W. Gubler, Heights in Diophantine geometry, volume 4 of New Mathematical Monographs, Cambridge University Press, Cambridge, 2006.

[CLZ09] P. CoRvajA, A. Levin, AND U. ZAnnier, Integral points on threefolds and other varieties, Tohoku Math. J. (2), 61:4 (2009), pp. 589-601.

[CZ02] P. CoRvajA AND U. ZANNIER, A subspace theorem approach to integral points on curves, C. R. Math. Acad. Sci. Paris, 334:4 (2002), pp. 267-271.

[CZ03] P. CORVAJA AND U. ZANNIER, On the number of integral points on algebraic curves, J. Reine Angew. Math., 565 (2003), pp. 27-42.

[CZ04a] P. Corvaja And U. Zannier, On a general Thue's equation, Amer. J. Math., 126:5 (2004), pp. 1033-1055.

[CZ04b] P. Corvaja AND U. ZANnier, On integral points on surfaces, Ann. of Math. (2), 160:2 (2004), pp. 705-726.

[CZ06a] P. Corvaja And U. ZANnIER, Addendum to: "On a general Thue's equation" [Amer. J. Math. 126 (2004), no. 5, 1033-1055], Amer. J. Math., 128:4 (2006), pp. 1057-1066.

[CZ06b] P. CoRvajA AND U. ZanNier, On the integral points on certain surfaces, Int. Math. Res. Not., 2006 (2006), pp. 1-20.

[EF02] J.-H. EverTSE AND R. FERRETTI, Diophantine inequalities on projective varieties, Int. Math. Res. Not., 25 (2002), pp. 1295-1330.

[EF08] J.-H. Evertse AND R. FERRETTI, A generalization of the Subspace Theorem with polynomials of higher degree, in "Diophantine approximation", volume 16 of Dev. Math., pp. 175-198. SpringerWienNewYork, Vienna, 2008.

[Fer00] R. FERRETT, Mumford's degree of contact and Diophantine approximations, Compositio Math., 121:3 (2000), pp. 247-262.

[FW94] G. FAltings And G. WÜstholz, Diophantine approximations on projective spaces, Invent. Math., 116:1-3 (1994), pp. 109-138.

[Gre75] M. Green, Some Picard theorems for holomorphic maps to algebraic varieties, Amer. J. Math., 97 (1975), pp. 43-75.

[Har74] R. Hartshorne, Varieties of small codimension in projective space, Bull. Amer. Math. Soc., 80 (1974), pp. 1017-1032.

[Har77] R. Hartshorne, Algebraic geometry, Springer-Verlag, New York, 1977. Graduate Texts in Mathematics, No. 52.

[Lan83] S. LANG, Fundamentals of Diophantine geometry, Springer-Verlag, New York, 1983.

[Lan87] S. LANG, Introduction to complex hyperbolic spaces, Springer-Verlag, New York, 1987.

[Lar73] M. LARSEN, On the topology of complex projective manifolds, Invent. Math., 19 (1973), pp. 251-260.

[Laz04] R. LAZARsfeld, Positivity in algebraic geometry. I, volume 48 of "Ergebnisse der Mathematik und ihrer Grenzgebiete. 3. Folge. A Series of Modern Surveys in Mathematics", Springer-Verlag, Berlin, 2004.

[Lev09] A. Levin, Generalizations of Siegel's and Picard's theorems, Ann. of Math. (2), 170:2 (2009), pp. 609-655.

[Mum77] D. Mumford, Stability of projective varieties, L'Enseignement Mathématique, Geneva, 1977. Lectures given at the "Institut des Hautes Études Scientifiques", Bures-surYvette, March-April 1976, Monographie de l'Enseignement Mathématique, No. 24.

[Ogu73] A. OGus, Local cohomological dimension of algebraic varieties, Ann. of Math. (2), 98 (1973), pp. 327-365.

[Ru97] M. Ru, On a general form of the second main theorem, Trans. Amer. Math. Soc., $349: 12$ (1997), pp. 5093-5105.

[Ru04] M. Ru, A defect relation for holomorphic curves intersecting hypersurfaces, Amer. J. Math., 126:1 (2004), pp. 215-226.

[Ru09] M. Ru, Holomorphic curves into algebraic varieties, Ann. of Math. (2), 169:1 (2009), pp. $255-267$.

[Voj87] P. VoJTA, Diophantine approximations and value distribution theory, volume 1239 of "Lecture Notes in Mathematics", Springer-Verlag, Berlin, 1987.

[Voj89] P. Vojta, A refinement of Schmidt's subspace theorem, Amer. J. Math., 111:3 (1989), pp. $489-518$.

[Voj97] P. VoJTA, On Cartan's theorem and Cartan's conjecture, Amer. J. Math., 119:1 (1997), pp. $1-17$.

[Wan04] J. T.-Y. WANG, An effective Schmidt's subspace theorem over function fields, Math. Z., 246:4 (2004), pp. 811-844. 
G. HEIER AND M. RU 